\title{
High-Temperature Ferrimagnetism with Large Coercivity and Exchange Bias in the Hexagonal Partially Ordered 3d/5d Perovskite $\mathrm{Ba}_{2} \mathrm{Fe}_{1.12} \mathrm{Os}_{0.88} \mathrm{O}_{6}$
}

Hai L. Feng, ${ }^{1 *}$ Peter Adler, ${ }^{1 *}$ Manfred Reehuis, ${ }^{2}$ Walter Schnelle, ${ }^{1}$ Philip Pattison,,${ }^{3,4}$ Andreas Hoser, ${ }^{2}$ Claudia Felser ${ }^{1}$, Martin Jansen ${ }^{1}$

${ }^{1}$ Max Planck Institute for Chemical Physics of Solids, 01187 Dresden, Germany

${ }^{2}$ Helmholtz-Zentrum Berlin für Materialien und Energie, D-14109 Berlin, Germany

${ }^{3}$ Swiss-Norwegian Beamline (SNBL) at the European Synchrotron Radiation Facility (ESRF), BP 220, F-38042 Grenoble Cedex 9, France

${ }^{4}$ Laboratory of Crystallography, Swiss Federal Institute of Technology, BSP-Dorigny, CH1015 Lausanne, Switzerland

*E-mail: Hai.Feng@cpfs.mpg.de

*E-mail: adler@cpfs.mpg.de 


\begin{abstract}
Double perovskite oxides $A_{2} B B^{\prime} \mathrm{O}_{6}$ combining $3 d$ and $4 d$ or $5 d$ transition metal ions at the $B$ and $B$ ' sites feature a variety of magnetic and magneto-electric properties. Targeting $\mathrm{Ba}_{2} \mathrm{FeOsO}_{6}$ we synthesized powder samples of non-stoichiometric $\mathrm{Ba}_{2} \mathrm{Fe}_{1.12} \mathrm{Oss}_{0.88} \mathrm{O}_{6}$ by solidstate reaction from the oxides. The crystal structure was investigated by using synchrotron powder $x$-ray and powder neutron diffraction. In contrast to $\mathrm{Ca}_{2} \mathrm{FeOsO}_{6}$ and $\mathrm{Sr}_{2} \mathrm{FeOsO}_{6}$, the compound adopts the hexagonal $6 \mathrm{~L}$ perovskite structure (space group $P \overline{3} \mathrm{~m} 1$ ) with partial $\mathrm{Fe}$ Os order at both the edge-sharing $\mathrm{B}_{2} \mathrm{O}_{9}$ dimer and the corner sharing $\mathrm{BO}_{6}$ transition metal sites. Magnetization, neutron diffraction, and ${ }^{57} \mathrm{Fe}$ Mössbauer spectroscopy results show that $\mathrm{Ba}_{2} \mathrm{Fe}_{1.12} \mathrm{Os}_{0.88} \mathrm{O}_{6}$ develops ferrimagnetic order well above room temperature at $T_{\mathrm{C}} \sim 370 \mathrm{~K}$. The non-saturated magnetization curve at $2 \mathrm{~K}$ features a magnetic moment of $0.4 \mu_{\mathrm{B}}$ per formula unit at $7 \mathrm{~T}$ and a pronounced hysteresis with a coercive field of about $2 \mathrm{~T}$. Large exchange bias effects are observed when the magnetization curves are measured after field cooling. The peculiar magnetic properties of $\mathrm{Ba}_{2} \mathrm{Fe}_{1.12} \mathrm{Os}_{0.88} \mathrm{O}_{6}$ are attributed to an inhomogeneous magnetic state formed as a consequence of the atomic disorder. Our results indicate that hexagonal double-perovskite-related oxides are a promising class of compounds for finding new materials with potential applications as hard magnets or in the area of spintronics.
\end{abstract}




\section{Introduction}

Recently, double-perovskite oxides, $A_{2} B B^{\prime} \mathrm{O}_{6}$, with $3 d$ and $4 d / 5 d$ transition metal cations in $B$ and $B^{\prime}$ sites, respectively, have attracted great attention due to the half-metallic nature found in $\mathrm{Sr}_{2} \mathrm{FeMoO}_{6}$ and $\mathrm{Sr}_{2} \mathrm{FeReO}_{6}$, which show Curie temperatures $\left(T_{\mathrm{C}}\right)$ higher than $400 \mathrm{~K}$. ${ }_{3}^{3}$ The half-metallic state was argued to result from a generalized double exchange mechanism, in which the $3 d-t_{2 g}$ and $5 d(4 d)-t_{2 g}$ electrons play an important role in the highly spin-polarized conduction. ${ }^{4}$ Since their prospects for spintronic applications are very high, much experimental progress has been made on the synthesis of $3 d / 5 d(4 d)$ double-perovskite oxides and related materials not only with respect to high-temperature magnetic transitions but also to explore other novel properties. For example, a high-temperature ferrimagnetic transition at $635 \mathrm{~K}$ was found in a nearly half-metallic compound $\mathrm{Sr}_{2} \mathrm{CrReO}_{6}{ }^{5}$ Transition-metal-only doubleperovskites, like $\mathrm{Mn}_{2} \mathrm{BReO}_{6}(B=\mathrm{Fe}, \mathrm{Mn})^{6-8}$ are pointing to another route towards new magnetic and magnetoresistive materials.

The insulating double-perovskite $\mathrm{Sr}_{2} \mathrm{CrOsO}_{6}$ (space group: $R \overline{3} m$ ) synthesized in 2007 displays a ferrimagnetic transition temperature of $725 \mathrm{~K}$, which is the highest magnetic transition temperature among all the double-perovskite oxides. ${ }^{9-11}$ In 2014 , another $3 d-5 d$ hybrid high-temperature ferrimagnetic insulator $\mathrm{Ca}_{2} \mathrm{FeOsO}_{6}$ was reported. ${ }^{12,13}$ However, the generalized double-exchange mechanism is not suited to account for the high-temperature transitions in $\mathrm{Sr}_{2} \mathrm{CrOsO}_{6}$ and $\mathrm{Ca}_{2} \mathrm{FeOsO}_{6}$ because they both are insulators. ${ }^{14,15}$ In comparison to the isoelectronic double-perovskite $\mathrm{Sr}_{2} \mathrm{FeOsO}_{6},{ }^{16-18}$ which crystallizes in a tetragonal doubleperovskite structure (space group: $I 4 / \mathrm{m}$ ) and shows successive antiferromagnetic (AFM) transitions around $140 \mathrm{~K}$ and $67 \mathrm{~K}, \mathrm{Ca}_{2} \mathrm{FeOsO}_{6}$ crystallizes in a monoclinic variant (space group: $P 2_{1} / n$ ) and shows room temperature ferrimagnetism driven by structural distortions. ${ }^{12,13}$ As a characteristic structural feature of double-perovskites, there are interpenetrating $B$ and $B^{\prime}$ face-centered cubic (fcc) sublattices, and their complex magnetic properties are mainly determined by the interplay between the intra-sublattice and inter-sublattices superexchange interactions. ${ }^{19-22}$ The exchange interactions are determined by the orbital interactions involving the $\mathrm{Fe}^{3+}\left(t_{2 \mathrm{~g}}{ }^{3} e_{\mathrm{g}}{ }^{2}\right)$ and $\mathrm{Os}^{5+}\left(t_{2 \mathrm{~g}}{ }^{3}\right)$ ions which can be sensitively modified by cationic substitution ${ }^{20-22}$ as well as by high pressure. ${ }^{23}$ In addition, the role of spin-orbit coupling for the magnetic properties of $\mathrm{Os}^{5+}$ double-perovskites is a matter of discussion. ${ }^{24}$

The strikingly different magnetic properties between $\mathrm{Ca}_{2} \mathrm{FeOsO}_{6}$ and $\mathrm{Sr}_{2} \mathrm{FeOsO}_{6}$, which appear to be related to different degrees of octahedral tilting, have motivated us to investigate Fe-Os hybrid oxides with distinctly different crystal structures. If the larger $\mathrm{Ba}$ cation is 
introduced to the A-site of the double-perovskite, the compounds are expected to adopt 6layered (6L) hexagonal $\mathrm{BaTiO}_{3}$ related crystal structures, given the tolerance factor $t=$ $\left(r_{A}+r_{O}\right) /\left[2^{1 / 2}\left(r_{B / 2}+r_{B^{\prime}} / 2+r_{O}\right)\right]$ is larger than 1 . Here $r$ corresponds to the effective ionic radii of the ions involved. For example, the compounds $\mathrm{Ba}_{2} \mathrm{CoTeO}_{6}, \mathrm{Ba}_{2} \mathrm{CrMoO}_{6}, \mathrm{Ba}_{2} \mathrm{CoOsO}_{6}$, and $\mathrm{Ba}_{2} \mathrm{NiOsO}_{6}$ were reported to adopt $6 \mathrm{~L} \mathrm{BaTiO}_{3}$ related structures. ${ }^{25-28} \mathrm{Ba}_{2} \mathrm{CoTeO}_{6}$ shows an antiferroelectric transition near $280 \mathrm{~K}$ and an antiferromagnetic transition at $15 \mathrm{~K}^{25}$ $\mathrm{Ba}_{2} \mathrm{CrMoO}_{6}$ displays a spin-glass transition at $37 \mathrm{~K}^{27}$ When trying to synthesize $\mathrm{Ba}_{2} \mathrm{FeOsO}_{6}$, we obtained the non-stoichiometric phase $\mathrm{Ba}_{2} \mathrm{Fe}_{1.12} \mathrm{Os}_{0.88} \mathrm{O}_{6}$. Here, we report on its synthesis, crystal structure and magnetic properties. The synthesis of a similar compound $\mathrm{Ba}_{2} \mathrm{Fe}_{0.92} \mathrm{Oss}_{1.08} \mathrm{O}_{6}$ was reported by Gemmill et al.; however, magnetic properties were not investigated. ${ }^{29}$ We have found that the $6 \mathrm{~L} \mathrm{Ba}_{2} \mathrm{Fe}_{1.12} \mathrm{Os}_{0.88} \mathrm{O}_{6}$ shows a high-temperature ferrimagnetic transition at $T_{\mathrm{C}} \approx 370 \mathrm{~K}$, which is even higher than the $T_{\mathrm{C}}$ of $320-350 \mathrm{~K}$ of $\mathrm{Ca}_{2} \mathrm{FeOsO}_{6}$. To the best of our knowledge, the former is the first example of a $6 \mathrm{~L}$ perovskite oxide showing ferrimagnetism above room temperature. Most remarkably, the magnetization curves of $\mathrm{Ba}_{2} \mathrm{Fe}_{1.12} \mathrm{Os}_{0.88} \mathrm{O}_{6}$ feature large coercivity and exchange bias (EB) effects, similar to nearly compensated ferrimagnetic Heusler alloys. ${ }^{30,31}$ The EB effect, namely a shift of the magnetization curve after field cooling along the field axis, is of great importance for magnetic devices. Given their large chemical and structural flexibility in combination with their potential for showing magnetic order above room temperature, double perovskites and their relatives are promising for designing new magnetic materials.

\section{Experimental}

Polycrystalline samples of nominal composition $\mathrm{Ba}_{2} \mathrm{FeOsO}_{6}$ were synthesized by solidstate reaction of the binary oxides $\mathrm{BaO}_{2}$ (Alfa Aesar, anhydrous, 84\% min), $\mathrm{Fe}_{2} \mathrm{O}_{3}$ (Alfa Aesar, 99.9\%) and $\mathrm{OsO}_{2}$ (Alfa Aesar, 83\% Os min). $\mathrm{BaO}_{2}, \mathrm{Fe}_{2} \mathrm{O}_{3}$, and $\mathrm{OsO}_{2}$ were weighed with mole ratio 4:1:2, thoroughly mixed, and pressed into pellets, inside a glove box. Then the pellets were transferred into corundum crucibles, which were placed and sealed inside quartz ampules. " $\mathrm{Ba}_{2} \mathrm{FeOsO}_{6}$ " was obtained after sintering for 96 hours at $980{ }^{\circ} \mathrm{C}$ with one intermittent regrinding.

For our laboratory and synchrotron $x$-ray diffraction study small pieces of " $\mathrm{Ba}_{2} \mathrm{FeOsO}_{6}$ " were cut from the synthesized pellets and then ground to a fine powder. Purity and structural properties of " $\mathrm{Ba}_{2} \mathrm{FeOsO}_{6}$ " were checked using a Huber G670 camera (Guinier technique, $\lambda=$ $1.54056 \AA$ (Cu-K $\alpha_{1}$ radiation), diffraction range $10^{\circ} \leq 2 \theta \leq 100^{\circ}$ in steps of $\left.0.005^{\circ}\right)$. High- 
resolution synchrotron powder data were collected at $295 \mathrm{~K}$ on the beam line BM01A (SwissNorwegian) at the ESRF in Grenoble ( $\lambda=0.6975 \AA$, glass capillary of diameter $0.3 \mathrm{~mm}$ ) using a Pilatus2M area detector. ${ }^{32}$ Rietveld refinements of the powder diffraction data were carried out with the program FullProf, using the atomic scattering factors provided by this program. ${ }^{33}$ A scanning electron microscope (SEM, Philips XL30) with an attached energy-dispersive $x$-ray spectrometer (EDX) was used for elemental analysis.

Powder neutron diffraction experiments were carried out on the instrument E9 at the BER II reactor of the Helmholtz-Zentrum Berlin, using a Ge-monochromator selecting the neutron wavelength $\lambda=1.7973 \AA$. On this instrument powder patterns of " $\mathrm{Ba}_{2} \mathrm{FeOsO}_{6}$ ” were collected at the temperatures $2,50,100,150,200,250,300,350$ and $400 \mathrm{~K}$ at the diffraction angles $8^{\circ} \leq$ $2 \theta \leq 141^{\circ}$. The refinements of the crystal and magnetic structures were carried out with the program FullProf applying the nuclear scattering lengths $b(\mathrm{O})=5.805 \mathrm{fm}, b(\mathrm{Fe})=9.54 \mathrm{fm}$, $b(\mathrm{Ba})=5.25 \mathrm{fm}$, and $b(\mathrm{Os})=11.0 \mathrm{fm} .{ }^{34}$ The magnetic form factors of the $\mathrm{Fe}^{3+}$ and $\mathrm{Os}^{5+}$ ions were taken from Refs. 35 and 36.

Using pieces of the obtained pellets, the electrical resistivity $(\rho)$ was measured with inverting direct current of $0.1 \mathrm{~mA}$ by a four-point method (PPMS, Quantum Design). Electrical contacts were made with Au wires and conducting silver paste.

Temperature and field-dependent magnetization measurements were performed with a magnetometer system (MPMS 3, Quantum Design) in applied magnetic fields of up to $\mu_{0} H=$ 7 T. In temperature-dependent experiments the sample was first cooled without magnetic field down to $2 \mathrm{~K}$. Subsequently, $M(T)$ data were obtained during heating (zero field cooled, ZFC) curve. Afterwards, a field cooled (FC) curve was recorded on cooling in the measuring field. Isothermal magnetization curves $M(H)$ as a function of applied field were obtained after cooling in zero field to the target temperature. After each measurement, the sample was heated to 400 $\mathrm{K}$, which is above the Curie temperature. Similarly, $M(H)$ curves were obtained after cooling in a magnetic field $H_{\mathrm{CF}}$. Magnetization curves at $2 \mathrm{~K}$ were measured after cooling in $\mu_{0} H_{\mathrm{CF}}=$ $\pm 1, \pm 2, \pm 4$, and $\pm 7 \mathrm{~T}$. After reaching the target temperature, the field was set as quick as possible to $+7 \mathrm{~T}$ and the demagnetization curve was measured, subsequently the field was ramped up from $-7 \mathrm{~T}$ to $+7 \mathrm{~T}$ for recording the remaining segments of the magnetization curve. Additional $M(H)$ curves at $2 \mathrm{~K}$ were obtained for low cooling fields $\mu_{0} H_{\mathrm{CF}}=+0.1,+0.2$, and $+0.5 \mathrm{~T}$. For selected higher temperatures up to $300 \mathrm{~K}, M(H)$ curves were measured after zero field cooling and for $\mu_{0} H_{\mathrm{CF}}=+7 \mathrm{~T}$.

${ }^{57}$ Fe-Mössbauer spectra were collected at selected temperatures using a standard WissEl spectrometer operated in the constant acceleration mode $\left({ }^{57} \mathrm{Co} / \mathrm{Rh}\right.$ source $)$ and a Janis 
SHI 850-5 closed cycle refrigerator. About $20 \mathrm{mg}$ of " $\mathrm{Ba}_{2} \mathrm{FeOsO}_{6}$ " powder was mixed with boron nitride and homogeneously distributed in an acrylic glass sample container with an inner diameter of $13 \mathrm{~mm}$. All isomer shifts are given relative to $\alpha$-iron. The data were evaluated with the MossWinn program ${ }^{37}$ using the thin absorber approximation. Hyperfine field distributions were derived using the Hesse-Rübartsch method implemented in MossWinn.

\section{Results and discussion}

\section{Sample composition and crystal structure}

The crystal structure of nominal $\mathrm{Ba}_{2} \mathrm{FeOsO}_{6}$ was investigated by laboratory and synchrotron $x$-ray powder diffraction. Aside from the reflections of a minor $\mathrm{Ba}_{2} \mathrm{SiO}_{4}$ impurity, the powder patterns were indexed based on a hexagonal lattice with the lattice parameters $a \approx$ $5.74 \AA$, and $c \approx 14.08 \AA$, suggesting that " $\mathrm{Ba}_{2} \mathrm{FeOsO}_{6}$ " belongs to the hexagonal $6 \mathrm{~L}$ $\mathrm{Ba}_{2} \mathrm{NiOsO}_{6}{ }^{28}$ type of structure. Possible space groups are P63/mmc (No. 194), P6 6 mc (No. 186), and $P \overline{3} m 1$ (No. 164). ${ }^{25-29,38-40}$ Rietveld refinements were carried out in these space groups, where finally the space group $P \overline{3} \mathrm{~m} 1$ gave the best fit. The result of the Rietveld refinements of the synchrotron data of $\mathrm{Ba}_{2} \mathrm{FeOsO}_{6}$ is depicted in Figure 1. It is found that the $\mathrm{Ba}$ and $\mathrm{O}$ atoms fully occupy the Wyckoff positions Ba1 in $2 c(0,0, z), \mathrm{Ba} 2$ and $\mathrm{Ba} 3$ in $2 d(1 / 3,2 / 3, z), \mathrm{O} 1, \mathrm{O} 2$, and O3 in $6 i(x,-x, z)$. In contrast, the Wyckoff positions $1 a(0,0,0), 1 b(0,0,1 / 2)$, and $2 d(1 / 3,2 / 3, z)$ are partially occupied by Fe and Os atoms. During the refinements, the occupancies of the Fe/Os sites were set to be $\operatorname{occ}(\mathrm{Fe})+\operatorname{occ}(\mathrm{Os})=1$. Our refinements revealed that the sample contains more iron than osmium giving the formula $\mathrm{Ba}_{2} \mathrm{Fe}_{1.12(1)} \mathrm{Os}_{0.88(1)} \mathrm{O}_{6}$, which is consistent with the ratio, $\mathrm{Fe} / \mathrm{Os}=1.13 / 0.87$, obtained from $\mathrm{EDX}$ analysis. The formation of volatile $\mathrm{OsO}_{4}$ during the synthesis may be the reason for the loss of Os. The refinements resulted in a residual $R_{F}=$ 0.023 (defined as $R_{F}=\sum|| F_{\text {obs }}|-| F_{\text {calc }}|| \sum\left|F_{\text {obs }}\right|$ ). Detailed crystal structure data of $\mathrm{Ba}_{1.12} \mathrm{Fe}_{0.88} \mathrm{OsO}_{6}$ obtained from SXRD refinements are summarized in Table S1. In the present space group $P \overline{3} m 1$, there are four independent Fe/Os sites as shown in Figure 2. Because of the different scattering powers of iron and osmium atoms the occupancies of the sites could be well refined from the synchrotron data. The following ratios were obtained: Fe1/Os1 = 0.878(12)/0.122(12), Fe2/Os2 = 0.536(12)/0.446(12), Fe3/Os3 = 0.719(5)/0.281(5), Fe4/Os4 = $0.255(8) / 0.745(8)$. 


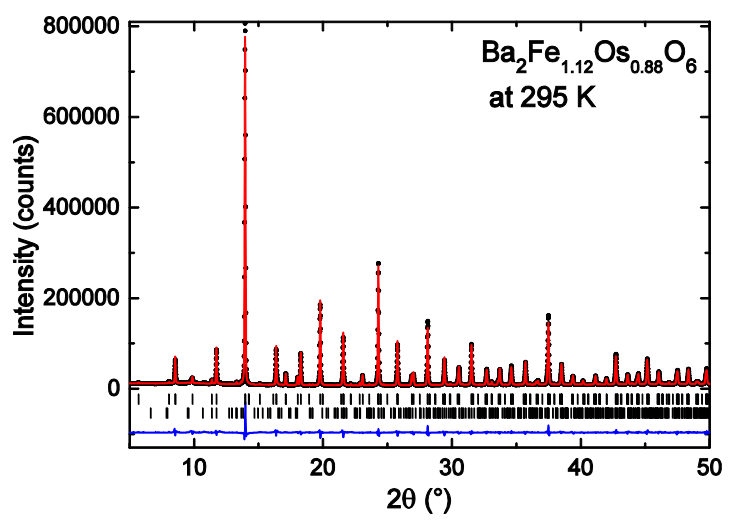

Figure 1. Results of the Rietveld refinements of the synchrotron powder diffraction data of $\mathrm{Ba}_{2} \mathrm{Fe}_{1.12} \mathrm{Os}_{0.88} \mathrm{O}_{6}$ collected at $295 \mathrm{~K}$. The crystal structure was refined in the trigonal space group $P \overline{3} m 1$. The calculated patterns (red) are compared with the observed ones (black circles). The difference patterns (blue) as well as the positions (black bars) of the nuclear Bragg reflections of $\mathrm{Ba}_{2} \mathrm{Fe}_{1.12} \mathrm{Os}_{0.88} \mathrm{O}_{6}$ (above) and the minor impurity $\mathrm{Ba}_{2} \mathrm{SiO}_{4}$ (below) are shown.

In order to determine the oxygen positions with good accuracy, the crystal structure of $\mathrm{Ba}_{2} \mathrm{Fe}_{1.12} \mathrm{Os}_{0.88} \mathrm{O}_{6}$ was also refined from powder neutron diffraction data collected in the range from 2 to $400 \mathrm{~K}$. Because to the fact that the neutron scattering lengths of Fe and Os differ only slightly, it was not possible to determine their occupancies from our powder neutron diffraction study. Therefore, we used the occupancies obtained from the synchrotron powder data, keeping them fixed during the Rietveld refinements. Further, we used the positional and thermal parameters obtained from the synchrotron study as starting model. At first, we refined the crystal structure from a data set collected at $400 \mathrm{~K}$, in the paramagnetic phase well above the ferrimagnetic ordering, which sets in at $T_{\mathrm{C}} \approx 370(10) \mathrm{K}$. The crystal structure was successfully refined in the space group $P \overline{3} m 1$ resulting in a residual $R_{F}=0.036$. Very similar results have been obtained from the data set collected at $2 \mathrm{~K}$ in the ferrimagnetically ordered state. The results are summarized in Tables 1 and S2. This shows that the magnetic ordering does not significantly change the structural parameters. Remarkably, variation of the occupancies of the oxygen atoms did not reveal significant deviations from 1, indicating the absence of significant oxygen deficiencies. As the Mössbauer spectra show only $\mathrm{Fe}^{3+}$ signals (see below), it is expected that the non-stoichiometry leads to a minority $\mathrm{Os}^{6+}$ component in addition to the majority $\mathrm{Os}^{5+}$ component. 
Table 1. Crystallographic data obtained from refinements of powder neutron diffraction data of $\mathrm{Ba}_{2} \mathrm{Fe}_{1.12} \mathrm{Os}_{0.88} \mathrm{O}_{6}$ at different temperatures.

\begin{tabular}{lll}
\hline & $2 \mathrm{~K}$ & $400 \mathrm{~K}$ \\
\hline space group & $P \overline{3} m 1$ (No. 164) & $P \overline{3} m 1$ (No. 164) \\
lattice parameters & $a=b=5.72931(14) \AA$, & $a=b=5.74823(15) \AA$, \\
& $c=14.0547(5) \AA$, & $c=14.1035(6) \AA$, \\
& $\alpha=\beta=90^{\circ}, \gamma=120^{\circ}$ & $\alpha=\beta=90^{\circ}, \gamma=120^{\circ}$ \\
cell volume & $399.54(3) \AA^{3}$ & $403.58(3) \AA^{3}$ \\
$R_{F}$ & 0.033 & 0.036 \\
\hline
\end{tabular}

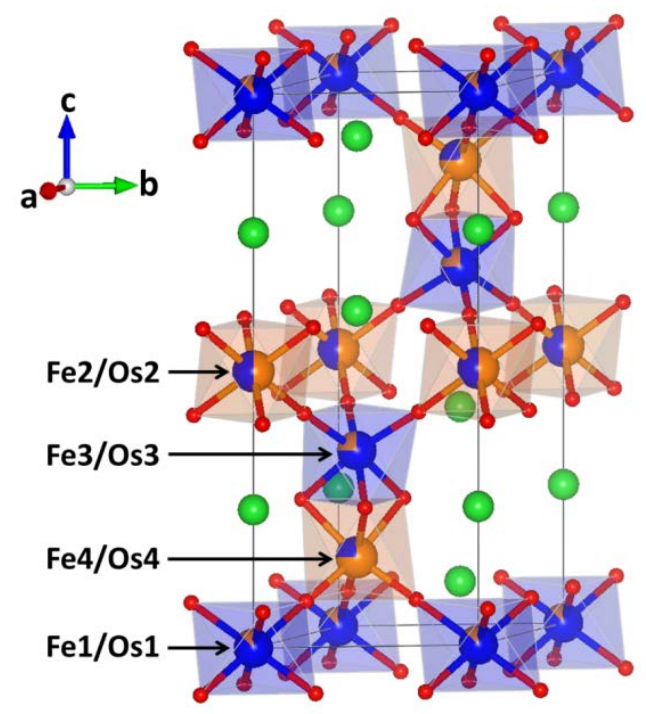

Figure 2. The $6 \mathrm{~L}$ perovskite structure of $\mathrm{Ba}_{2} \mathrm{Fe}_{1.12} \mathrm{Os}_{0.88} \mathrm{O}_{6}$. $\mathrm{Ba}, \mathrm{Fe}, \mathrm{Os}$, and $\mathrm{O}$ atoms are represented by green, blue, orange, and red spheres, respectively.

A view of the crystal structure of $\mathrm{Ba}_{2} \mathrm{Fe}_{1.12} \mathrm{Os}_{0.88} \mathrm{O}_{6}$ is shown in Figure 2. The main structural motives are face-sharing $\mathrm{M}_{2} \mathrm{O}_{9}$ dimeric units which are connected by single cornersharing $\mathrm{MO}_{6}$ units. Basically, the crystal structure corresponds to that of $\mathrm{Ba}_{2} \mathrm{NiOsO}_{6}$, but all the transition metal sites are partially occupied. The Fe3/Os3-centered octahedra are predominantly occupied by Fe, while for the Fe4/Os4-centered octahedra, Os is prevailing. They constitute the sets of face sharing octahedra, with the Fe3/Os3-Fe4/Os4 separation of 2.719(12) $\AA$ across the shared face. The Fe3/Os3-centered and Fe4/Os4-centered octahedra connect to the Fe2/Os2- 
centered and Fe1/Os1-centered octahedra, respectively, by corner sharing. While the Fe1/Os1 sites are predominantly occupied by Fe atoms, nearly equal amounts of Fe and Os atoms are found at the Fe2/Os2 sites. Thus, the excess of iron atoms compared to stoichiometric $\mathrm{Ba}_{2} \mathrm{FeOsO}_{6}$ are mainly located at the corner-sharing sites. Selected bond lengths and bond angles of $\mathrm{Ba}_{2} \mathrm{Fe}_{1.12} \mathrm{Oss}_{0.88} \mathrm{O}_{6}$ obtained from powder neutron refinement at 2 and $400 \mathrm{~K}$ are summarized in Table 2. At $400 \mathrm{~K}$, the bond angles of the $\mathrm{Fe} 4 / \mathrm{Os} 4-\mathrm{O} 2-\mathrm{Fe} 1 / \mathrm{Os} 1$ and Fe3/Os3-O3-Fe2/Os2 (corner-sharing octahedra) are 179.4 and $175.9^{\circ}$, respectively, while the bond angle of Fe3/Os3-O1-Fe4/Os4 (face-sharing octahedra) is 84.5 . In Table 2, it can be seen that for Fe1/Os1, Fe2/Os2, and Fe3/Os3 the metal-oxygen bond distances vary at $400 \mathrm{~K}$ between 1.987 and $2.016 \AA$ A. However, the Fe4/Os4-centered (osmium rich) octahedra shows a pronounced distortion: the bond lengths of Fe4/Os4-O1 and Fe4/Os4-O2 are 2.060 and 1.913 Å respectively. Large octahedral distortions have been reported for hexagonal perovskites with $\mathrm{Os}_{2} \mathrm{O}_{9}$ dimeric units such as $\mathrm{K}_{3} \mathrm{NaOs}_{2} \mathrm{O}_{9}$ and $\mathrm{Ba}_{3} \mathrm{NaOs}_{2} \mathrm{O}_{9}{ }^{41,42}$ and were attributed to displacement of the high-valent osmium ions from the center positions. The mean value of the bond distances of Fe4/Os4-O1 and Fe4/Os4-O2 is $1.987 \AA$, which is close to the values obtained for the other three metal sites.

A similar compound $\mathrm{Ba}_{2} \mathrm{Fe}_{0.92} \mathrm{Os}_{1.08} \mathrm{O}_{6}$ was reported to crystallize in the space group ${ }^{P 6}{ }_{3} m c{ }^{29}$ In comparison with the space group $P \overline{3} \mathrm{~m} 1$, there are three independent Fe/Os sites, where the Fe1/Os1 and Fe2/Os2 sites merge into one Wyckoff position. A distortion of the Os rich face-sharing octahedra was observed too. However, in the case of $\mathrm{Ba}_{2} \mathrm{Fe}_{1.12} \mathrm{Oss}_{0.88} \mathrm{O}_{6}$ the unequal site occupation factors require the descent in space group symmetry. In contrast to the prototype $\mathrm{Ba}_{2} \mathrm{NiOsO}_{6}$ the present sample of $6 \mathrm{~L} \mathrm{Ba}_{2} \mathrm{Fe}_{1.12} \mathrm{Os}_{0.88} \mathrm{O}_{6}$ shows only partial Fe-Os ordering. Depending on sample preparation also the monoclinic $\mathrm{Ca}_{2} \mathrm{FeOsO}_{6}{ }^{12,13}$ and tetragonal $\mathrm{Sr}_{2} \mathrm{FeOsO}_{6}{ }^{16-18}$ double-perovskites revealed a certain degree of Fe-Os antisite disorder; however, the disorder seems to be enhanced in $6 \mathrm{~L} \mathrm{Ba}_{2} \mathrm{Fe}_{1.12} \mathrm{Os}_{0.88} \mathrm{O}_{6}$. Attempts to improve the degree of atomic order by varying the synthesis conditions have not been successful, so far. 
Table 2. Selected bond lengths and bond angles from powder neutron refinements of $\mathrm{Ba}_{2} \mathrm{Fe}_{1.12} \mathrm{Os}_{0.88} \mathrm{O}_{6}$ patterns at 2 and $400 \mathrm{~K}$.

\begin{tabular}{lll}
\hline Bond length $(\AA)$ & $2 \mathrm{~K}$ & $400 \mathrm{~K}$ \\
\hline Fe1/Os1-O2 & $2.047(6)$ & $2.016(8) \times 6$ \\
Fe2/Os2-O3 & $1.993(6)$ & $2.012(6) \times 6$ \\
Fe3/Os3-O1 & $1.966(10)$ & $1.987(13) \times 3$ \\
Fe3/Os3-O3 & $2.007(7)$ & $1.988(8) \times 3$ \\
Fe4/Os4-O1 & $2.088(12)$ & $2.060(13) \times 3$ \\
Fe4/Os4-O2 & $1.855(8)$ & $1.913(9) \times 3$ \\
Bond angle $\left(^{\circ}\right)$ & & \\
Fe1/Os1-O2-Fe4/Os4 & $176.6(5)$ & $179.4(5)$ \\
Fe2/Os2-O3-Fe3/Os3 & $176.0(4)$ & $175.9(5)$ \\
Fe3/Os3-O1-Fe4/Os4 & $84.1(2)$ & $84.5(3)$ \\
\hline
\end{tabular}

\section{Electrical transport}

The electrical resistivity $\rho(T)$ of a sintered polycrystalline pellet of $\mathrm{Ba}_{2} \mathrm{Fe}_{1.12} \mathrm{Os}_{0.88} \mathrm{O}_{6}$ is shown in Figure 3a. The sample is insulating, with a resistivity increasing by several orders of magnitude as the temperature decreases, and exceeds the measurement limit for temperatures lower than $120 \mathrm{~K}$. Attempts were made to plot the data on $T^{-1}$ and $T^{-1 / 4}$ scales as shown in Figure $3 \mathrm{~b}$. The resistivity is found to be roughly linear on a $T^{-1 / 4}$ scale, in accordance with a three-dimensional variable range hopping transport model. Similar behavior has been reported for other osmium double-perovskites such as $\mathrm{Ca}_{2} \mathrm{CoOsO}_{6}{ }^{43}$ 

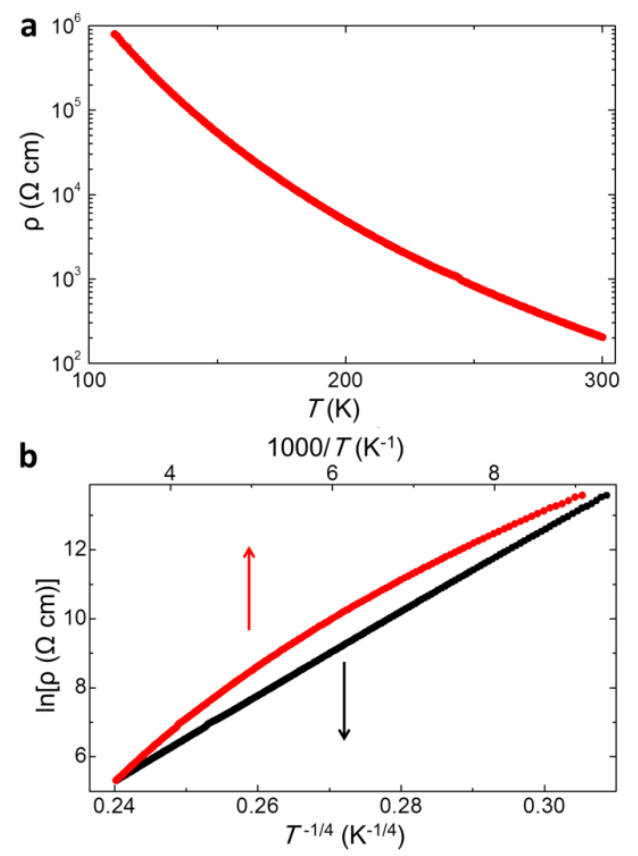

Figure 3. (a) Temperature dependence of the electrical resistivity of $\mathrm{Ba}_{2} \mathrm{Fe}_{1.12} \mathrm{Os}_{0.88} \mathrm{O}_{6}$, and (b) the corresponding $\ln (\rho)$ vs. $T^{-1}$ and $\ln (\rho)$ vs. $T^{-1 / 4}$ curves.

\section{Magnetic properties: Exchange bias}

The temperature dependence of the magnetic susceptibility $\chi=M / H$ of $\mathrm{Ba}_{2} \mathrm{Fe}_{1.12} \mathrm{Os}_{0.88} \mathrm{O}_{6}$ with applied fields of $\mu_{0} H=0.1 \mathrm{~T}$ and $1 \mathrm{~T}$ is shown in Figure 4 . The magnetic susceptibility increases below $\approx 370 \mathrm{~K}$ with cooling and the typical field dependence indicates a ferro- or ferrimagnetic transition. The rather gradual increase in susceptibility suggests that the magnetic state is not completely homogeneous.

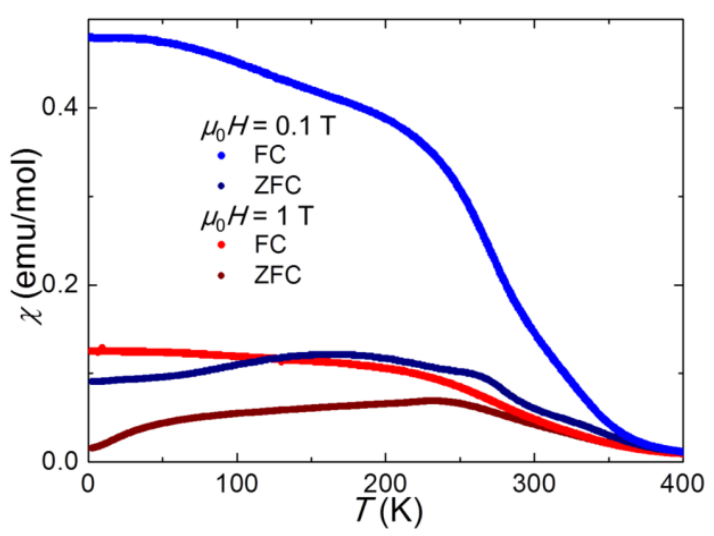

Figure 4. Temperature dependence of the magnetic susceptibility of $\mathrm{Ba}_{2} \mathrm{Fe}_{1.12} \mathrm{Os}_{0.88} \mathrm{O}_{6}$. Shown are the susceptibilities measured in field (FC) and zero-field cooling (ZFC) processes at the indicated magnetic fields. 
To further investigate the nature of this transition, the isothermal magnetization $M(H)$ of $\mathrm{Ba}_{2} \mathrm{Fe}_{1.12} \mathrm{Os}_{0.88} \mathrm{O}_{6}$ was measured between 2 and $400 \mathrm{~K}$ after zero-field cooling as shown in Figure 5. A linear $M(H)$ curve without hysteresis is found for $\mathrm{Ba}_{2} \mathrm{Fe}_{1.12} \mathrm{Os}_{0.88} \mathrm{O}_{6}$ for $T=400 \mathrm{~K}$, indicating the paramagnetic state. The isothermal magnetization curves at $300 \mathrm{~K}$ and below show ferromagnetic behavior with hysteresis. However, the magnetization is not saturated even at $2 \mathrm{~K}$ under $7 \mathrm{~T}$ and the magnetization of $0.42 \mu_{\mathrm{B}} / \mathrm{f}$.u. is much lower than the expected spinonly saturation value $M_{\mathrm{S}}$ of $8.0 \mu_{\mathrm{B}} / \mathrm{f}$.u. obtained by assuming that $\mathrm{Fe}^{3+}\left(t_{2 \mathrm{~g}}{ }^{3} e_{\mathrm{g}}{ }^{2}\right)$ and $\mathrm{Os}^{5+}\left(t_{2 \mathrm{~g}}{ }^{3}\right)$ were ferromagnetically coupled. It is more reasonable to consider the transition at $\approx 370 \mathrm{~K}$ as a ferrimagnetic transition, which would result in a saturation magnetization of $2.0 \mu_{\mathrm{B}} /$ f.u. The large difference between the observed magnetization of $0.42 \mu_{\mathrm{B}} / \mathrm{f}$.u. and the spin-only value of $M_{\mathrm{S}}=2.0 \mu_{\mathrm{B}} / \mathrm{f}$.u. can be largely attributed to the considerable $B$-site disorder (as discussed in the crystal structure part). Displacement of the $\mathrm{Fe}^{3+}$ ions introduces AFM interactions between the displaced $\mathrm{Fe}^{3+}$ and the regular $\mathrm{Fe}^{3+}$ ions. Accordingly, the $B$-site disorder reduces the saturation magnetization, which has been described by the mathematical expression $M_{\mathrm{S}}=M_{\mathrm{ST}} \times(1-2 \times$ AS) $\mu_{\mathrm{B}} /$ f.u., where $M_{\mathrm{ST}}$ is the theoretical saturation magnetization without the presence of antisite disorder, and $A S$ is the degree of $B$-type antisites. ${ }^{44,45}$ In $\mathrm{Ba}_{2} \mathrm{Fe}_{1.12} \mathrm{Oss}_{0.88} \mathrm{O}_{6}$, the $M_{\mathrm{ST}}$ is $2.0 \mu_{\mathrm{B}} /$ f.u., and the $A S$ is $\approx 0.27$, thus resulting in the saturation magnetization value of 0.92 $\mu_{\mathrm{B}} / \mathrm{f}$.u. Furthermore, the excess $\mathrm{Fe}$ atoms will give rise to additional antiferromagnetic $\mathrm{Fe}-\mathrm{O}-\mathrm{Fe}$ couplings which also reduces the net magnetization. Most remarkably, the magnetic order occurs above room temperature in spite of the large number of antisite defects. Similar observations for other Fe-based double-perovskites ${ }^{46}$ have been attributed to the strong AF interactions between the $\mathrm{Fe}^{3+}$ centers. A remarkable feature of the $M(H)$ curve of $\mathrm{Ba}_{2} \mathrm{Fe}_{1.12} \mathrm{Os}_{0.88} \mathrm{O}_{6}$ is the high coercive field with $\mu_{0} H_{\mathrm{C}}$ of up to $2 \mathrm{~T}$ at $T=2 \mathrm{~K}$, which declines to $0.2 \mathrm{~T}$ at $T=300 \mathrm{~K}$. Different batches of samples reveal a certain variation in $\mu_{0} H_{\mathrm{C}}(1.7-2.0$ $\mathrm{T}$ ) which may reflect small variations in the Fe/Os rati and the degree of antisite disorder. 


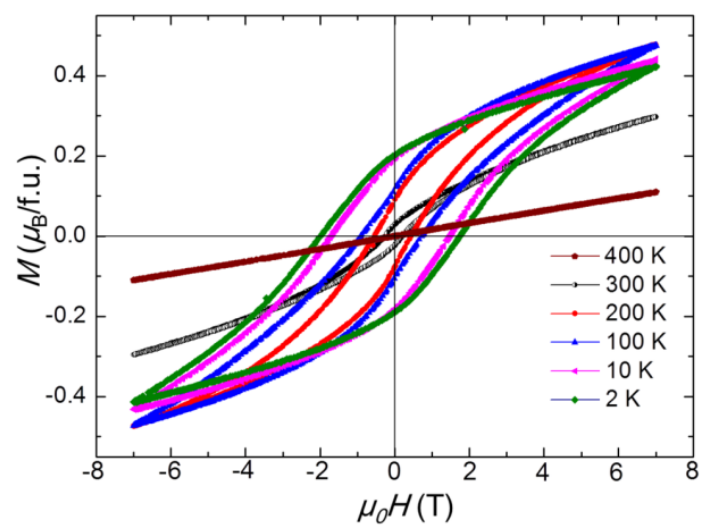

Figure 5. The isothermal magnetization of $\mathrm{Ba}_{2} \mathrm{Fe}_{1.12} \mathrm{Os}_{0.88} \mathrm{O}_{6}$ measured at the indicated temperatures.

Recently. there has been considerable interest in exchange bias (EB) effects in ferrimagnetic materials. ${ }^{30,31}$ The EB effect will result in a shift of the $M(H)$ curve along the magnetic field axis if the magnetization measurements are performed after cooling in a field $H_{\text {CF. The }}$ EB effect was first observed in nanoparticles composed of a ferromagnetic (FM) cobalt core and an antiferromagnetic (AFM) cobalt oxide shell and explained in terms of a unidirectional exchange anisotropy induced by the AFM/FM interface. ${ }^{47}$ In order to investigate whether $\mathrm{Ba}_{2} \mathrm{Fe}_{1.12} \mathrm{Oss}_{0.88} \mathrm{O}_{6}$ shows EB behavior we have measured a selection of $M(H)$ curves after cooling in various $H_{\mathrm{CF}}$ and at different temperatures $T$. A representative result is shown in Figure 6a. It is obvious that $\mathrm{Ba}_{2} \mathrm{Fe}_{1.12} \mathrm{Os}_{0.88} \mathrm{O}_{6}$ shows large EB effects, indeed. For instance, after cooling in $\mu_{0} H_{\mathrm{CF}}=+7 \mathrm{~T}$, the $M(H)$ curve at $T=2 \mathrm{~K}$ is shifted in the negative field direction, whereas it is shifted in the positive field direction for $\mu_{0} H_{\mathrm{CF}}=-7 \mathrm{~T}$. Further, a stretching and shifting of the curve in the $+M$ or $-M$ direction is found. From the $M(H)$ curves the coercive fields $H_{\mathrm{C}}$ and exchange-bias fields $H_{\mathrm{EB}}$ were extracted according to $H_{\mathrm{C}}=\left|H_{1}-H_{2}\right| / 2$ and $H_{\mathrm{EB}}=$ $\left|H_{1}+H_{2}\right| / 2$, respectively. Here, $H_{1}$ and $H_{2}$ are the lower and upper magnetic fields, where the magnetization vanishes. Both, $H_{\mathrm{C}}$ and $H_{\mathrm{EB}}$, are larger for positive than for negative $H_{\mathrm{CF}}$ values and they decrease with decreasing $H_{\mathrm{CF}}$ (inset of Figure $6 \mathrm{~b}$ ). Even small cooling fields $\mu_{0} H_{\mathrm{CF}}$ of $0.1-0.5$ T are sufficient to induce a significant shift of the $M(H)$ curve. The EB effect persists at least up to $T=300 \mathrm{~K}$, however, $H_{\mathrm{C}}$ as well as $H_{\mathrm{EB}}$ decrease strongly with increasing temperature (Figure 6b). 

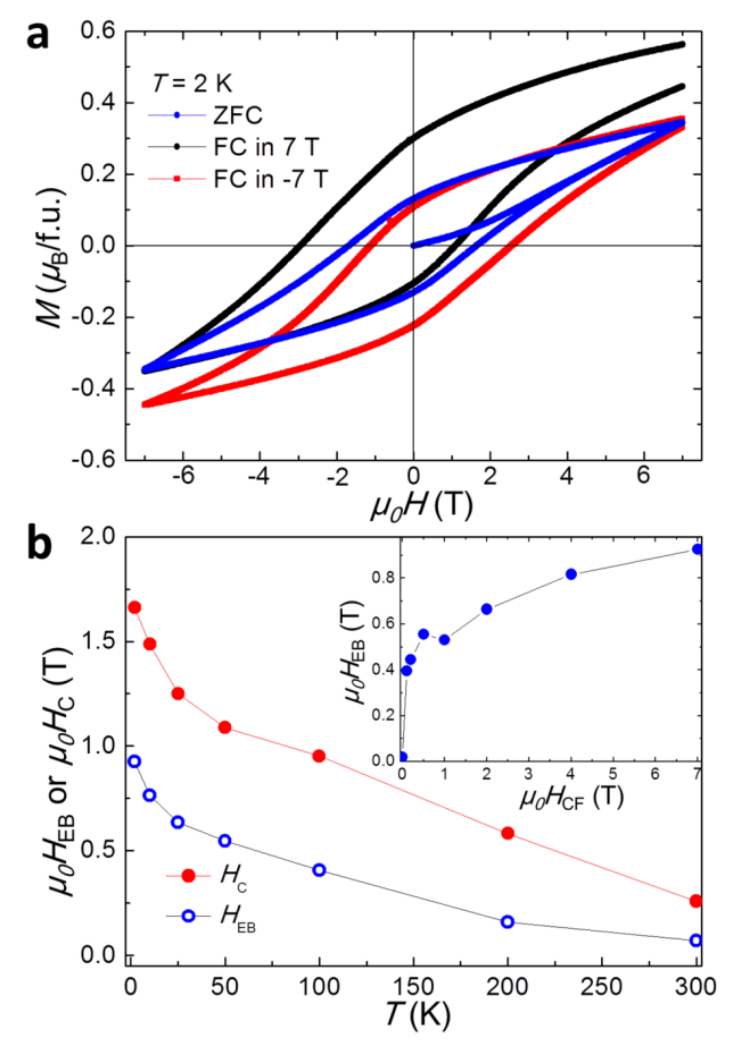

Figure 6. (a) $M(H)$ loops of $\mathrm{Ba}_{2} \mathrm{Fe}_{1.12} \mathrm{Os}_{0.88} \mathrm{O}_{6}$ at $2 \mathrm{~K}$ measured with $\mathrm{ZFC}$ and FC modes. (b) Temperature dependence of coercive field $\left(H_{\mathrm{C}}\right)$ determined from ZFC mode and exchange bias field $\left(H_{\mathrm{EB}}\right)$ of $\mathrm{Ba}_{2} \mathrm{Fe}_{1.12} \mathrm{Os}_{0.88} \mathrm{O}_{6}$ obtained after cooling in $H_{\mathrm{CF}}=+7 \mathrm{~T}$. The inset shows the cooling field dependence of $H_{\mathrm{EB}}$.

Usually, EB effects occur at the interface of composite FM/AFM materials which is applied, for instance, in spin valves for pinning the spin direction of the fixed FM layer. While EB systems for technical applications are heterostructures, EB effects have been also observed in several structurally homogeneous systems, which revealed various combinations of magnetic phases. ${ }^{48}$ Atomic disorder seems to be a general feature, and among others, EB behavior was reported for spinglass related systems. Small EB effects $\left(\mu_{0} H_{\mathrm{EB}} \approx 0.03 \mathrm{~T}\right)$ in the ferromagnetic $3 d / 3 d$ DP oxide $\mathrm{LaSrCoMnO}_{6}$ were attributed to spin-glass-like ordering at low temperature. ${ }^{49}$ Recently, giant EB effects were reported for the nearly compensated ferrimagnetic Heusler alloys $\mathrm{Mn}_{3-x} \mathrm{Pt}_{x} \mathrm{Ga}$, where the huge $\mathrm{EB}$ effects were attributed to the presence of ferrimagnetic clusters in the compensated host. ${ }^{30}$ The huge EB effects were attributed to the presence of ferrimagnetic clusters in the compensated host, which are formed as a consequence of antisite disorder. Also zero-field cooled EB was observed for Heusler alloys. ${ }^{50}$ Similar to Heusler compounds, double-perovskite-related materials have different lattice sites for magnetic ions, too, and they are frequently prone to antisite disorder. The hexagonal $6 \mathrm{~L}$ perovskite structure 
is particularly interesting as it intrinsically provides two different lattice sites, namely those in the face-and corner-sharing octahedra, respectively. Atomic ordering in a hexagonal double perovskite leads to four magnetic sublattices. In the present material $\mathrm{Ba}_{2} \mathrm{Fe}_{1.12} \mathrm{Os}_{0.88} \mathrm{O}_{6}$ both Fe/Os nonstoichiometry and antisite disorder lead to a considerable reduction of the total magnetic moment as the moments of $\mathrm{Fe}^{3+}$ sitting at different crystallographic sites nearly cancel each other due to AFM $\mathrm{Fe}^{3+}-\mathrm{Fe}^{3+}$ interactions. We thus attribute the large coercivity and the EB effect in $\mathrm{Ba}_{2} \mathrm{Fe}_{1.12} \mathrm{Oss}_{0.88} \mathrm{O}_{6}$ to the coexistence of nearly compensated and ferrimagnetic regions on the microscale. In summary, $\mathrm{Ba}_{2} \mathrm{Fe}_{1.12} \mathrm{Os}_{0.88} \mathrm{O}_{6}$ is a ferrimagnetic oxide with high Curie temperature and large EB effects, which may provide further insights into the nature of EB effects in ferrimagnets and which encourages the search for useful magnetic materials in double-perovskite related oxides.

\section{Spin structure}

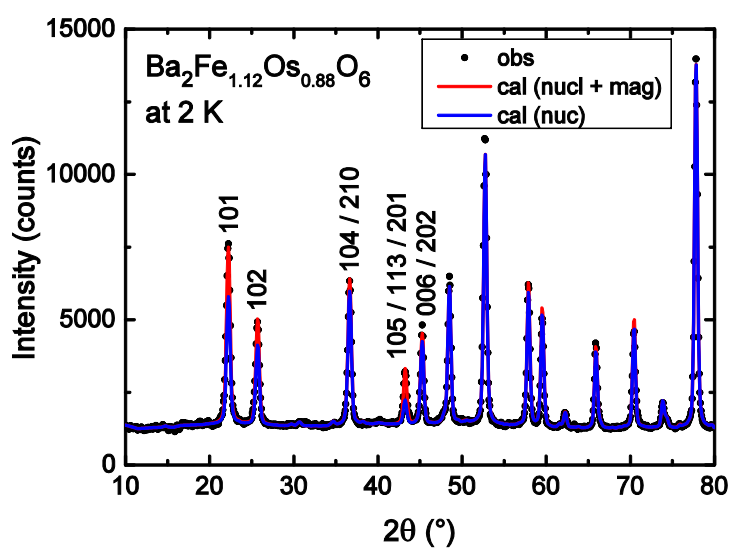

Figure 7. Powder neutron diffraction pattern of $\mathrm{Ba}_{2} \mathrm{Fe}_{1.12} \mathrm{Os}_{0.88} \mathrm{O}_{6}$ taken at $2 \mathrm{~K}$. The calculated pattern of the pure nuclear contribution (blue) as well as the sum of the nuclear and the magnetic contribution (red) is compared with the observed one (black circles).

In order to investigate the spin structure of $\mathrm{Ba}_{2} \mathrm{Fe}_{1.12} \mathrm{Os}_{0.88} \mathrm{O}_{6}$ we have collected a powder neutron pattern at $2 \mathrm{~K}$. In comparison to the data collected at $400 \mathrm{~K}$, it is apparent that magnetic intensities are observable at the positions of nuclear Bragg reflections. In Figure 7 it can be seen that the strongest magnetic intensities are noticeable at the positions of the reflections 102 at $25.6^{\circ}$, as well as at the positions of the overlapping reflections 101/003 at $22.1^{\circ}$ and $105 / 113 / 201$ at $43.1^{\circ}$, respectively. This confirms the presence of ferri- or ferromagnetism as 
found from magnetization measurements. For the refinements of the magnetic structure, we only used the data up to $2 \theta=50^{\circ}$, where the nuclear reflections contain a sufficient part of magnetic intensity. In our first model of magnetic ordering, we allowed the magnetic moments of the $\mathrm{Fe}$ and Os atoms to vary along the hexagonal $c$ axis. It was found that the magnetic moments at the sites Fe1/Os1 and Fe3/Os3 are much larger than those of the sites Fe2/Os2 and Fe4/Os4. Further, it has been shown that the moments of Fe1/Os1 and Fe3/Os3 are coupled antiparallel to those of $\mathrm{Fe} 2 / \mathrm{Os} 2$ and Fe4/Os4 suggesting the presence of an ordered ferrimagnetic state. However, it was not possible to determine the magnetic moments of the four metal sites independently with good accuracy. This can be ascribed to the fact that only a small number of reflections was available, where magnetic intensity could be clearly observed. Further, part of the reflections overlap. This finally led to a strong increase of standard deviations. However, our Mössbauer measurements (see below) indicate that all iron ions are in the valence state $\mathrm{Fe}^{3+}$ and carry similar magnetic moments at the four different Wyckoff positions. Therefore, in our refinement of the magnetic structure we constrained the magnetic moments of the $\mathrm{Fe}^{3+}$ ions as well as those of the $\mathrm{Os}^{5+}$ ions to be equal at all the sites. The total moment $\mu$ (Fei/Osi) $(i=1-4)$ at each site is then the sum of the $\mathrm{Fe}^{3+}$ and $\mathrm{Os}^{5+}$ moments weighted by the respective site occupancies. In this model, any possible $\mathrm{Os}^{6+}$ content due to nonstoichiometry was neglected. The refinement of the moments $\left[\mu_{\exp }\left(\mathrm{Fe}^{3+}\right)=4.0(2) \mu_{\mathrm{B}}\right.$, and $\left.\mu_{\exp }\left(\mathrm{Os}^{5+}\right)=1.5(2) \mu_{\mathrm{B}}\right]$ resulted in a satisfactory residual $R_{\mathrm{M}}=0.058$ (defined as $R_{\mathrm{M}}=\sum|| I_{\mathrm{obs}} \mid-$ $\left.\left|I_{\text {calc }}\right|\left|/ \sum\right| I_{\text {obs }} \mid\right)$. This way, we were able to follow the temperature dependence of the magnetic moments of the $\mathrm{Fe}^{3+}$ ions and $\mathrm{Os}^{5+}$ ions up to the Curie temperature of about $375 \mathrm{~K}$ (Figure 8), which is in good agreement with the results of the magnetization measurements. Figure 8 also shows the temperature dependence of the lattice parameters. A slight anomaly may occur close to the magnetic transition into the ferrimagnetic state. The magnetic moment of the $\mathrm{Fe}^{3+}$ ions at $2 \mathrm{~K}$ is comparable to that of other $\mathrm{Fe}^{3+}$ oxides, but somewhat smaller than the theoretical moment of $\mu_{\exp }=5.0 \mu_{\mathrm{B}}$ for the $\mathrm{Fe}^{3+} 3 d^{5}$ high-spin state. The magnetic moment is also in accord with the Mössbauer spectra. Accordingly, neglecting non-stoichiometry, the Os ions should be in the state $\mathrm{Os}^{5+}\left(5 d^{3}\right.$ state, $\left.t_{2 \mathrm{~g}}{ }^{3}\right)$, where one expects a spin-only moment of $\mu_{\exp }=3.0 \mu_{\mathrm{B}}$. But interestingly, we found for the osmium a strongly reduced moment $\mu_{\exp }=1.5(2) \mu_{\mathrm{B}}$. This value is quite similar as for double-perovskites $\mathrm{Sr}_{2} \mathrm{BOsO}_{6}$ with a diamagnetic $B^{3+}$ ion, where typically $\mathrm{Os}^{5+}$ moments between 1.6 and $2 \mu_{\mathrm{B}}$ were found from powder neutron diffraction studies. ${ }^{51,52}$ By contrast, an unambiguous determination of the Os moments from neutron data of the hybrid $3 d / 5 d$ systems $\mathrm{Sr}_{2} \mathrm{FeOsO}_{6}$ and $\mathrm{Ca}_{2} \mathrm{FeOsO}_{6}$ turned out to be difficult. ${ }^{13,17} \mathrm{X}$-ray magnetic circular dichroism studies suggested an osmium moment of $2.5 \mu_{\mathrm{B}}$ for $\mathrm{Ca}_{2} \mathrm{FeOsO}_{6}{ }^{23}$ 
We also considered a second model of magnetic ordering, where we assumed that the magnetic moments of $\mathrm{Ba}_{2} \mathrm{Fe}_{1.12} \mathrm{Os}_{0.88} \mathrm{O}_{6}$ are aligned within the hexagonal $a b$ plane. For this model, the magnetic intensity is additionally generated on the reflection 003 , which practically lies at the same position as the reflection 101. This refinement resulted in an enlarged ratio $I(101 / 003) / I(102)=2.584$. However, for the first model, where the moments are aligned parallel to the $c$ axis, the ratio resulted in the value 2.306. Because of the fact that this value is only slightly smaller than the observed one of 2.313 we can conclude that the first model is correct.

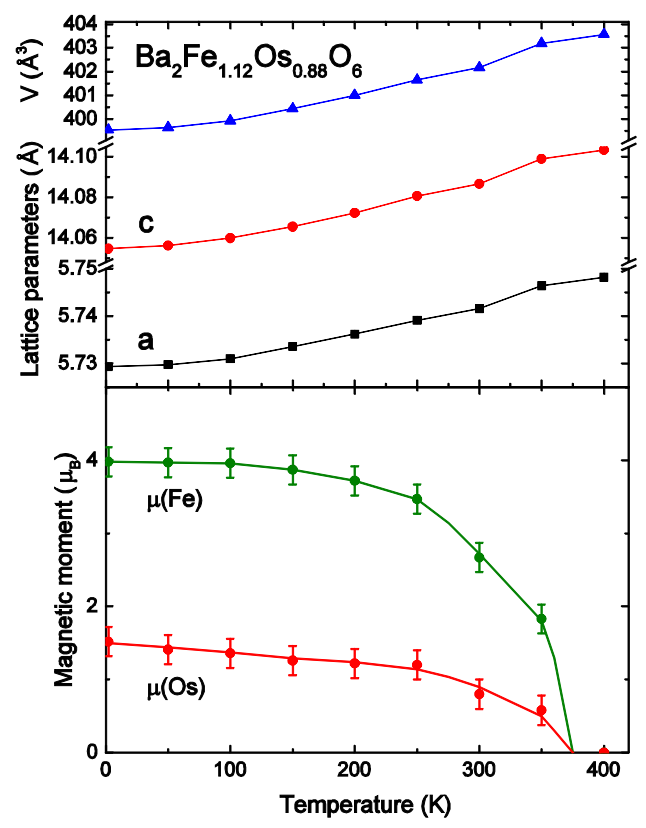

Figure 8. Temperature dependence of the lattice parameters (top) and the magnetic moments of the Fe and Os atoms (bottom).

Our magnetic measurements on $\mathrm{Ba}_{2} \mathrm{Fe}_{1.12} \mathrm{Os}_{0.88} \mathrm{O}_{6}$ already indicated that the magnetic moments of the Fe and Os atoms are ordered in a ferrimagnetic state. The neutron diffraction experiments revealed that the magnetic moments of the four sublattices Fe1/Os1, Fe2/Os2, Fe3/Os3, and Fe4/Os4 show the spin directions +-+- (Figure 9). Because of the different moment values at each site, one finds in total a saturation magnetization in agreement with a ferrimagnetic state. Taking into account the multiplicities of the Wyckoff positions we finally obtained $M_{\mathrm{s}}=\mu(\mathrm{Fe} 1 / \mathrm{Os} 1)-\mu(\mathrm{Fe} 2 / \mathrm{Os} 2)+2 \mu(\mathrm{Fe} 3 / \mathrm{Os} 3)-2 \mu(\mathrm{Fe} 4 / \mathrm{Os} 4)=3.55 \mu_{\mathrm{B}} / 3 \mathrm{f} . \mathrm{u}$, which corresponds to $1.2 \mu_{\mathrm{B}} / \mathrm{fu}$. This value is still larger than the magnetization of $0.42 \mu_{\mathrm{B}} / \mathrm{f}$.u. at $7 \mathrm{~T}$ found from our magnetization measurements. However, in Figure 5 it can be seen that the 
magnetization up to $7 \mathrm{~T}$ is still not saturated which points to the presence of a strong single-ion anisotropy. Therefore, we can assume that only the ordered moments, which are randomly aligned parallel to the field direction, show a spontaneous magnetization. Thus, the magnetization and neutron diffraction data are reasonably compatible with each other, in particular if also the approximations inherent in the evaluation of the neutron data of this partially ordered system are kept in mind.

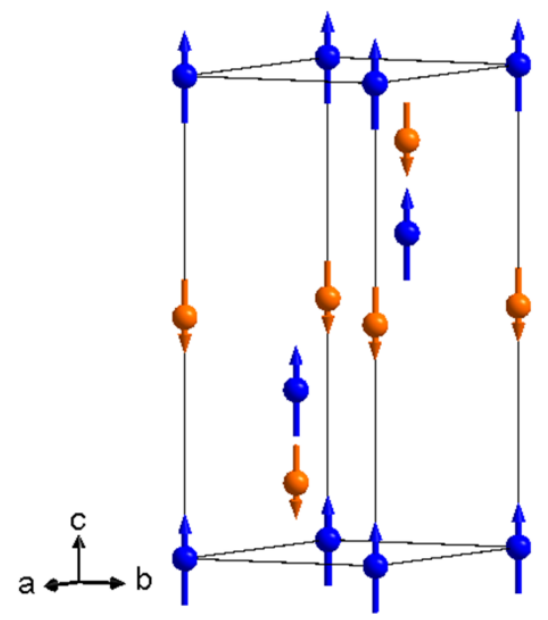

Figure 9. Illustration of the ferrimagnetic spin structure of $\mathrm{Ba}_{2} \mathrm{Fe}_{1.12} \mathrm{Oss}_{0.88} \mathrm{O}_{6}$. Blue and orange spheres/ arrows correspond to iron- and osmium-dominated sites, respectively (c.f. Figure 2).

\section{Mössbauer spectroscopy}

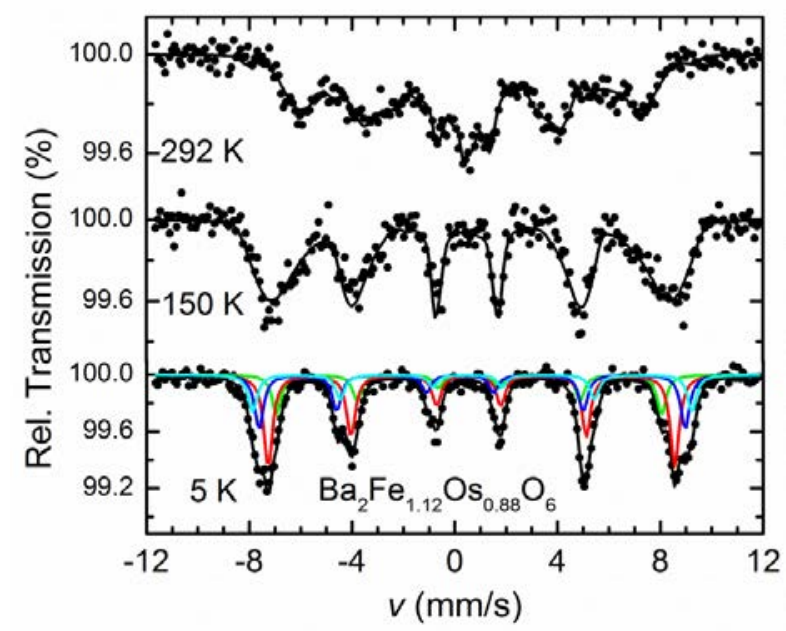

Figure 10. ${ }^{57} \mathrm{Fe}$ Mössbauer spectra of $\mathrm{Ba}_{1.12} \mathrm{Fe}_{0.88} \mathrm{OsO}_{6}$ at the indicated temperatures. Solid black and colored lines correspond to the calculated total spectrum and the subspectra, respectively, dots to the experimental spectrum. 
While the diffraction techniques average over larger sample volumes Mössbauer spectroscopy provides information on the local structural and magnetic properties. In Figure $10{ }^{57} \mathrm{Fe}$ Mössbauer spectra of $\mathrm{Ba}_{2} \mathrm{Fe}_{1.12} \mathrm{Os}_{0.88} \mathrm{O}_{6}$ at different temperatures are shown. Because of the high electronic absorption of Os and $\mathrm{Ba}$, the resonance effect is small and thus the statistics of the spectra is poor. Nevertheless, it is evident that the spectra reveal magnetic hyperfine splitting at all temperatures, which confirms that the Curie temperature is above room temperature. At low temperature ( $5 \mathrm{~K}$ ) the spectrum features some structure, in contrast to the single six-line pattern observed for the tetragonal double-perovskite $\mathrm{Sr}_{2} \mathrm{FeOsO}_{6}{ }^{17,53}$ However, the spectrum is poorly resolved, which points to the superposition of several inequivalent Fe sites. Roughly the spectrum can be described by two strongly broadened sextets with relative intensities of $60 \%$ and $40 \%$, respectively. Taking into account the excess of iron at the cornersharing sites in $\mathrm{Ba}_{2} \mathrm{Fe}_{1.12} \mathrm{Os}_{0.88} \mathrm{O}_{6}$, this remarkably corresponds to the ratio of Fe ions located in edge-sharing and corner-sharing sites, and thus, it is tempting to associate the subspectra with these sites. An improved fit is obtained by using four inequivalent sites where the area fractions are roughly compatible with the Fe occupancies at the four inequivalent sites of the $P \overline{3} m 1$ crystal structure as derived from the synchrotron XRD data. However, as the lines are still broadened this fit is not unambiguous. The Mössbauer parameters resulting from the fit are summarized in Table 3. The isomers shifts IS as well as the hyperfine fields $B_{\mathrm{hf}}$ are within the range of values which is typical for $\mathrm{Fe}^{3+}$ (high spin $t_{2 \mathrm{~g}}{ }^{3} \mathrm{eg}^{2}$ configuration). In particular, both, IS and $B_{\mathrm{hf}}$ for the majority component are close to the values of $\mathrm{Sr}_{2} \mathrm{FeOsO}_{6}{ }^{17,53}$ Considering the relative intensities and the site occupancies (Tables S1 and 3) this subspectrum is attributed to the Fe atoms residing at the face-sharing $2 d$ site. The spectrum confirms that all iron sites are fully magnetically ordered at $5 \mathrm{~K}$, but the variation in $B_{\mathrm{hf}}$ for the different components suggests that the individual moments differ slightly at the various Fe sites. The hyperfine field values of 46-53 $\mathrm{T}$ are in agreement with the large magnetic moments on the Fe-dominated positions as derived from the neutron diffraction data (Figure 7). There is no evidence for any iron sites with oxidation states different from +3 . At higher temperatures, the spectra become even broader and they cannot be reliably fit by a superposition of discrete sextets. Instead, a hyperfine field distribution reproduces approximately the shape of the spectra, where, however, single isomer shift and quadrupole splitting parameters had to be used. The very broad features occurring over an extended temperature range suggest that due to the atomic disorder also the magnetic state features considerable disorder, and actually, rather a distribution of Curie temperatures than a well-defined value are expected. This is in agreement with the observation that the $B_{\mathrm{hf}}$ distribution at $292 \mathrm{~K}$ extends down to $0 \mathrm{~T}$, indicating that a fraction of about $10 \%$ of the $\mathrm{Fe}$ 
atoms is already in the paramagnetic state. These considerations provide also an explanation for the very gradual rise of the susceptibility below $370 \mathrm{~K}$ (Figure 4) and support our interpretation of the exchange bias effect.

Table 3. Results from fitting the Mössbauer spectrum of $\mathrm{Ba}_{2} \mathrm{Fe}_{1.12} \mathrm{Os}_{0.88} \mathrm{O}_{6}$ at $5 \mathrm{~K}$ by four hyperfine sextets using the thin absorber approximation and the perturbation limit ( $Q S<<B_{\mathrm{hf}}$ ). Here, IS, $B \mathrm{hf}, Q S$, and $\Gamma$ correspond to the isomer shift, hyperfine field, quadrupole splitting parameter, and linewidth, respectively. In the last column the relative area fraction $A$ is given. The linewidths were constrained to be the same for all components. The colors corresponding to the respective subspectra in Figure 10 are given in parenthesis.

\begin{tabular}{lccccc}
\hline component & $\begin{array}{c}I S \\
\left(\mathrm{~mm} \mathrm{~s}^{-1}\right)\end{array}$ & $\begin{array}{c}B_{\mathrm{hf}} \\
(\mathrm{T})\end{array}$ & $\begin{array}{c}\text { QS } \\
\mathrm{mm} \mathrm{s}^{-1}\end{array}$ & $\begin{array}{c}\Gamma \\
\mathrm{mm} \mathrm{s}^{-1}\end{array}$ & $\begin{array}{c}A \\
(\%)\end{array}$ \\
\hline 1 (green) & $0.54(2)$ & $46.6(2)$ & $0.02(3)$ & $0.42(2)$ & $18(2)$ \\
2 (red) & $0.59(1)$ & $49.2(1)$ & $0.11(2)$ & 0.42 & $41(2)$ \\
3 (blue) & $0.44(2)$ & $51.6(2)$ & $0.47(2)$ & 0.42 & $24(2)$ \\
4 (cyan) & $0.59(2)$ & $53.2(2)$ & $0.19(4)$ & 0.42 & $17(2)$ \\
\hline \hline
\end{tabular}

\section{Magnetic exchange interactions}

Considering first a stoichiometric $6 \mathrm{~L}$ perovskite $\mathrm{Ba}_{2} \mathrm{FeOsO}_{6}$ with perfect atomic order one expects that the magnetism is mainly determined by pronounced superexchange interactions between $\mathrm{Fe}^{3+}\left(t_{2 \mathrm{~g}}{ }^{3} e_{\mathrm{g}}{ }^{2}\right)$ and $\mathrm{Os}^{5+}\left(t_{2 \mathrm{~g}}{ }^{3}\right)$ ions via the corner-sharing octahedra. In our real material the corresponding bond angles are only slightly deviating from $180^{\circ}$, suggesting strong exchange interactions via this pathway. The metal-metal distance of $2.7 \AA$ within the face-sharing $\mathrm{M}_{2} \mathrm{O}_{9}$ dimers is too large for direct metal-metal interactions, and the superexchange interactions via the $M-\mathrm{O}-M$ pathways with bond angles somewhat smaller than $90^{\circ}$ are expected to be much weaker than those via the corner-sharing octahedra. According to the Goodenough-Kanamori rules it could be expected that the $\mathrm{Fe}^{3+}-\mathrm{Os}^{5+}$ interactions are ferromagnetic. However, the ferromagnetic interaction involves a $\sigma$-exchange pathway with virtual charge transfer from the occupied Fe $e_{\mathrm{g}}$ orbitals into the empty Os $e_{\mathrm{g}}$ orbitals. ${ }^{23}$ In case of the $5 d$ element osmium, these orbitals are, however, considerably higher in energy than in corresponding $3 d$ transition metal oxides, which make this pathway much weaker. Therefore, rather the antiferromagnetic $\pi$-exchange pathway via the half-occupied $t_{2 \mathrm{~g}}{ }^{3}$ shells of $\mathrm{Fe}^{3+}$ and 
$\mathrm{Os}^{5+}$ may dominate the magnetic properties and give rise to the frequently observed ferrimagnetic behavior. The $\sigma$-exchange pathway is particularly sensitive to octahedral tilting and the different spin structures of $\mathrm{Ca}_{2} \mathrm{FeOsO}_{6}$ and $\mathrm{Sr}_{2} \mathrm{FeOsO}_{6}$ have been traced back to the larger degree of tilting in the former case which suppresses the $\sigma$-exchange. ${ }^{13}$ However, also the detailed energy position of the empty $e_{\mathrm{g}}$ orbital of $\mathrm{Os}^{5+}$ was suggested to be of importance. Thus, a destabilization of the $\mathrm{Os}^{5+} e_{\mathrm{g}}$ orbital is believed to reduce the strength of the ferromagnetic interaction in $\mathrm{Sr}_{2} \mathrm{FeOsO}_{6}$ under high pressure which rationalizes the pressureinduced appearance of a ferrimagnetic state in this material ${ }^{23}$ adopting antiferromagnetic spin structures at ambient pressure. Nevertheless, the tetragonal crystal structure of $\mathrm{Sr}_{2} \mathrm{FeOsO}_{6}$ is retained under pressure.

In the present $\mathrm{Ba}_{2} \mathrm{FeOsO}_{6}$ system the bond angle is only slightly reduced from $180^{\circ}$ and thus ferromagnetic $\mathrm{Fe}^{3+}-\mathrm{Os}^{5+}$ coupling cannot be ruled out a priori. However, our experimental results clearly evidence ferrimagnetic order which suggests that the $\mathrm{Fe}^{3+}-\mathrm{Os}^{5+}$ interactions are strongly antiferromagnetic and thus the $\pi$-superexchange pathway is dominant. Because of nonstoichiometry and antisite disorder the magnetism of $\mathrm{Ba}_{2} \mathrm{Fe}_{1.12} \mathrm{Os}_{0.88} \mathrm{O}_{6}$ will be different compared to stoichiometric and well-ordered $\mathrm{Ba}_{2} \mathrm{FeOsO}_{6}$ since antiferromagnetic $\mathrm{Fe}^{3+}-\mathrm{O}-\mathrm{Fe}^{3+}$ exchange interactions reduce the ordered magnetic moments as discussed above. Theoretical studies on the electronic structure and magnetic exchange interactions are required to obtain a deeper insight into the magnetic properties of hexagonal $\mathrm{Ba}_{2} \mathrm{FeOsO}_{6}$. Further on, the influence of the atomic disorder on the magnetic properties remains to be investigated in detail.

\section{Conclusions}

Aiming at the preparation of $\mathrm{Ba}_{2} \mathrm{FeOsO}_{6}$ we have obtained non-stoichiometric $\mathrm{Ba}_{2} \mathrm{Fe}_{1.12} \mathrm{Os}_{0.88} \mathrm{O}_{6}$ in bulk powder form and investigated its properties. Our structural studies with synchrotron powder x-ray and neutron diffraction show that this compound adopts the hexagonal 6L perovskite structure with partial Fe-Os order at both, the edge-sharing dimer, and single corner sharing transition metal sites. In spite of the considerable degree of atomic disorder, $\mathrm{Ba}_{2} \mathrm{Fe}_{1.12} \mathrm{Os}_{0.88} \mathrm{O}_{6}$ shows ferrimagnetic order well above room temperature $\left(T_{\mathrm{C}} \sim 370\right.$ $\mathrm{K})$, but the total ordered moment is reduced due to antiferromagnetic $\mathrm{Fe}^{3+}-\mathrm{Fe}^{3+}$ interactions resulting from disorder and non-stoichiometry. The magnetization curve at $2 \mathrm{~K}$ shows a large coercive field of up to $2 \mathrm{~T}$ as well as large exchange bias effects which are related to the inhomogeneous magnetic state formed in course of the atomic disorder. Our results suggest that 
hexagonal double-perovskite-related oxides are a promising class of compounds where materials for potential applications as hard magnets or as spintronic materials may be found. In contrast to other recently reported ferrimagnetic $3 d / 5 d$ perovskites like $\mathrm{Pb}_{2} \mathrm{FeOsO}_{6}\left(T_{\mathrm{C}} \approx 280\right.$ $\mathrm{K})^{54}$ and the $A$-site ordered quadrupole perovskite $\mathrm{CaCu}_{3} \mathrm{Fe}_{2} \mathrm{Os}_{2} \mathrm{O}_{12}\left(T_{\mathrm{C}} \approx 580 \mathrm{~K}\right),{ }^{55}$ which had to be stabilized at extreme pressure conditions, the present hexagonal perovskite can be prepared in larger quantities at ambient pressure. By varying the composition, by changing the degree of atomic order or by imposing epitaxial strain in thin films it may be possible to get even higher $T_{\mathrm{C}}$ 's and to modify the exchange bias in hexagonal $3 d / 5 d$ perovskites.

\section{ASSOCIATED CONTENT}

\section{Supporting Information}

Supplemental data: the refined SXRD data at room temperature (Table S1); the refined PND data at 400 and $2 \mathrm{~K}$ (Table S2).

\section{AUTHOR INFORMATION}

\section{Corresponding Authors}

*Hai L. Feng and Peter Adler

E-mail: hai.feng@cpfs.mpg.de (HLF) and adler@cpfs.mpg.de (PA)

Notes

The authors declare no competing financial interest.

\section{Acknowledgment}

This research was partially supported by the Deutsche Forschungsgemeinschaft through SFB 1143. C.F. acknowledges support by the Joint Initiative for Research and Innovation within the Fraunhofer and Max Planck cooperation program. We thank R. Koban, H. Borrmann, and U. Burkhardt for performing magnetic, X-ray, and EDX measurements. The authors are grateful to the Swiss-Norwegian Beamline consortium for providing access to synchrotron radiation. 


\section{REFERENCES}

(1) Kobayashi, K. L.; Kimura, T.; Sawada, H.; Terakura, K.; Tokura, Y. Room-Temperature Magnetoresistance in an Oxide Material with an Ordered Double-Perovskite Structure. Nature 1998, 395, 677-680.

(2) Tomioka, Y.; Okuda, T.; Okimoto, Y.; Kumai, R.; Kobayashi, K. I.; Tokura, Y. Magnetic and Electronic Properties of a Single Crystal of Ordered Double Perovskite $\mathrm{Sr}_{2} \mathrm{FeMoO}_{6}$. Phys. Rev. B 2000, 61, 422-427.

(3) Kobayashi, K. I.; Kimura, T.; Tomioka, Y.; Sawada, H.; Terakura, K.; Tokura, Y. Intergrain Tunneling Magnetoresistance in Polycrystals of the Ordered Double Perovskite $\mathrm{Sr}_{2} \mathrm{FeReO}_{6}$. Phys. Rev. B 1999, 59, 11159-11162.

(4) Erten, O.; Meetei, O. N.; Mukherjee, A.; Randeria, M.; Trivedi, N.; Woodward, P. Theory of Half-Metallic Ferrimagnetism in Double Perovskites. Phys. Rev. Lett. 2011, 107, 257201.

(5) Kato, H.; Okuda, T.; Okimoto, Y.; Tomioka, Y.; Takenoya, Y.; Ohkubo, A.; Kawasaki, M.; Tokura, Y. Metallic Ordered Double-Perovskite $\mathrm{Sr}_{2} \mathrm{CrReO}_{6}$ with Maximal Curie Temperature of 635 K. Appl. Phys. Lett. 2002, 81, 328-330.

(6) Aréval-López, A. M.; McNally, G. M.; Attfield, J. P. Large Magnetization and Frustration Switching of Magnetoresistance in the Double-Perovskite Ferrimagnet $\mathrm{Mn}_{2} \mathrm{FeReO}_{6}$. Angw. Chem. Int. Ed. 2015, 54, 12074-12077.

(7) Li, M. R.; Retuerto, M.; Deng, Z.; Stephens, P. W.; Croft, M.; Huang, Q.; Wu, H.; Deng, X.; Kotliar, G.; Sánchez-Benitez, J.; Hadermann, J.; Walker, D.; Greenblatt, M. Giant Magnetoresistance in the Half-Metallic Double-Perovskite Ferrimagnet $\mathrm{Mn}_{2} \mathrm{FeReO}_{6}$. Angew. Chem. Int. Ed. 2015, 54, 12069-12073.

(8) Li, M. R.; Hodges, J. P.; Retuerto, M.; Deng, Z.; Stephens, P. W.; Croft, M. C.; Deng, X.; Kotliar, G.; Sánchez-Benitez, J.; Walker, D.; Greenblatt, M. $\mathrm{Mn}_{2} \mathrm{MnReO}_{6}$ : Synthesis and Magnetic Structure Determination of a New Transition-Metal-Only Double Perovskite Canted Antiferromagnet. Chem. Mater. 2016, 28, 3148-3158.

(9) Krockenberger, Y.; Mogare, K.; Reehuis, M.; Tovar, M.; Jansen, M.; Vaitheeswaran, G.; Kanchana, V.; Bultmark, F.; Delin, A.; Wilhelm, F.; Rogalev, A.; Winkler, A.; Alff, L. $\mathrm{Sr}_{2} \mathrm{CrOsO}_{6}$ : End Point of a Spin-Polarized Metal-Insulator Transition by $5 d$ Band Filling. Phys. Rev. B 2007, 75, 020404.

(10) Morrow, R.; Soliz, J. R.; Hauser, A. J.; Gallagher, J. C.; Susner, M. A.; Sumption, M. D.; Aczel, A. A.; Yan, J.; Yang, F.; Woodward, P. M. The Effect of Chemical Pressure on the 
Structure and Properties of $A_{2} \mathrm{CrOsO}_{6}(A=\mathrm{Sr}$, Ca) Ferrimagnetic Double Perovskite. $J$. Solid State Chem. 2016, 238, 46-52.

(11) Krockenberger, Y.; Reehuis, M.; Tovar, M.; Mogare, K. M.; Jansen, M.; Alff, L. A Neutron Scattering Study of the Crystal and Magnetic Structure of $\mathrm{Sr}_{2} \mathrm{CrOsO}_{6}$. J. Mag. Mater. 2007, 310, 1854-1856.

(12) Feng, H. L.; Arai, M.; Matsushita, Y.; Tsujimoto, Y.; Guo, Y. F.; Sathish, C. I.; Wang, X.; Yuan, Y. H.; Tanaka, M.; Yamaura, K. High-Temperature Ferrimagnetism Driven by Lattice Distortion in Double Perovskite $\mathrm{Ca}_{2} \mathrm{FeOsO}_{6}$. J. Am. Chem. Soc. 2014, 136, 33263329.

(13) Morrow, R.; Freeland, J. W.; Woodward, P. M. Probing the Links between Structure and Magnetism in $\mathrm{Sr}_{2-\mathrm{x}} \mathrm{Ca}_{\mathrm{x}} \mathrm{FeOsO}_{6}$ Double Perovskites. Inorg. Chem. 2014, 53, 7983-7992.

(14) Meetei, O. N.; Erten, O.; Randeria, M.; Trivedi, N.; Woodward, P. Theory of High Tc Ferrimagnetism in a Multiorbital Mott Insulator. Phys. Rev. Lett. 2013, 110, 087203.

(15) Das, H.; Sanyal, P.; Saha-Dasgupta, T.; Sarma, D. D. Origin of Magnetism and Trend in Tc in Cr-Based Double Perovskites: Interplay of Two Driving Mechanisms. Phys. Rev. B 2011, 83, 104418.

(16) Feng, H. L.; Tsujimoto, Y.; Guo, Y. F.; Sun, Y.; Sathish, C. I.; Yamaura, K. High Pressure Synthesis, Crystal Structure, and Magnetic Properties of the Double-Perovskite $\mathrm{Sr}_{2} \mathrm{FeOsO}_{6}$. High Press. Res. 2013, 33, 221-228.

(17) Paul, A. K.; Reehuis, M.; Ksenofontov, V.; Yan, B.; Hoser, A.; Többens, D. M.; Abdala, P. M.; Adler, P.; Jansen, M.; Felser, C. Lattice Instability and Competing Spin Structures in the Double Perovskite Insulator $\mathrm{Sr}_{2} \mathrm{FeOsO}_{6}$. Phys. Rev. Lett. 2013, 111, 167205.

(18) Paul, A. K.; Jansen, M.; Yan, B.; Felser, C.; Reehuis, M.; Abdala, P. M. Synthesis, Crystal Structure, and Physical Properties of $\mathrm{Sr}_{2} \mathrm{FeOsO}_{6}$. Inorg. Chem. 2013, 52, 6713-6719.

(19) Feng, H. L.; Schnelle, W.; Tjeng, L. H.; Jansen, M. Synthesis, Crystal Structures, and Magnetic Properties of Double Perovskites $\mathrm{SrLaNiOsO}_{6}$ and $\mathrm{BaLaNiOsO}_{6}$. Solid State Commun. 2016, 243, 49-54.

(20) Morrow, R.; Yan, J.; McGuire, M. A.; Freeland, J. W.; Haskel, D.; Woodward, P. M. Effects of Chemical Pressure on the Magnetic Ground States of the Osmate Double Perovskites $\mathrm{SrCaCoOsO}_{6}$ and $\mathrm{Ca}_{2} \mathrm{CoOsO}_{6}$. Phys. Rev. B 2015, 92, 094435.

(21) Wang, H.; Zhu, S.; Ou, X.; Wu, H. Ferrimagnetism in the Double Perovskite $\mathrm{Ca}_{2} \mathrm{FeOsO}_{6}$ : A Density Functional Study. Phys. Rev. B 2014, 90, 054406.

(22) Hou, Y. S.; Xiang, H. J.; Gong, X.G. Lattice-Distortion Induced Magnetic Transition from Low-Temperature Antiferromagnetism to High-Temperature Ferrimagnetism in 
Double Perovskites $A_{2} \mathrm{FeOsO}_{6}(A=\mathrm{Ca}, \mathrm{Sr})$. Sci. Rep. 2015, 5, 13159.

(23) Veiga, L. S. J.; Fabbris, G.; Van Veenendaal, M.; Souza-Neto, N. M.; Feng, H. L.; Yamaura, K.; Haskel, D. Fragility of Ferromagnetic Double Exchange Interactions and Pressure Tuning of Magnetism in 3d-5d Double Perovskite $\mathrm{Sr}_{2} \mathrm{FeOsO}_{6}$. Phys. Rev. B 2015, 91, 235135.

(24) Taylor, A. E.; Morrow, R.; Fishman, R. S.; Calder, S.; Kolesnikov, A. I.; Lumsden, M. D.; Woodward, P. M.; Christianson, A. D. Spin-Orbit Coupling Controlled Ground State in $\mathrm{Sr}_{2} \mathrm{ScOsO}_{6}$. Phys. Rev. B 2016, 93, 220408 (R).

(25) Ivanov, S. A.; Nordblad, P.; Mathieu, R.; Tellgren, R.; Ritter, C. Neutron Diffraction Studies and the Magnetism of an Ordered Perovskite: $\mathrm{Ba}_{2} \mathrm{CoTeO}_{6}$. Dalton Trans. 2010, 39, 5490-5499.

(26) Chanlert, P.; Kurita, N.; Tanaka, H.; Goto, D.; Matsuo, A.; Kindo, K. Field-driven Successive Phase Transitions in the Quasi-Two-Dimensional Frustrated Antiferromagnet $\mathrm{Ba}_{2} \mathrm{CoTeO}_{6}$ and Highly Degenerate Classical Ground States. Phys. Rev. B 2016, 93, 094420.

(27) Sher, F.; Paul Attfield, J. Synthesis, Structure and Magnetic Properties of $\mathrm{Ba}_{2} \mathrm{CrMoO}_{6}$. Solid State Sci. 2006, 8, 277-279.

(28) Treiber, U.; Kemmler-Sack, S. Investigations on Hexagonal 6L Perovskites $\mathrm{Ba}_{2} B^{\mathrm{II}} \mathrm{OsO}_{6}$ with $B^{\mathrm{II}}=$ Co, Ni. Z. anorg. allg. Chem. 1980, 470, 95-102.

(29) Gemmill, W. R.; Smith, M. D.; zur Loye, H.-C. Synthesis and Structural Characterization of two New Hexagonal Osmates: $\mathrm{Ba}_{2} \mathrm{Fe}_{0.92} \mathrm{Os}_{1.08} \mathrm{O}_{6}$ and $\mathrm{Ba}_{2} \mathrm{Co}_{0.77} \mathrm{Os}_{1.23} \mathrm{O}_{6}$. Solid State Sci. 2007, 9, 380-384.

(30) Nayak, A. K.; Nicklas, M.; Chadov, S.; Khuntia, P.; Shekhar, C.; Kalache, A.; Baenitz, M.; Skourski, Y.; Guduru, V. K.; Puri, A.; Zeitler, U.; Coey, J. M. D.; Felser, C. Design of Compensated Ferrimagnetic Heusler Alloys for Giant Tunable Exchange Bias. Nat. Mat. 2015, 14, 679-684.

(31) Sahoo, R.; Wollmann, L.; Selle, S.; Höche, T.; Ernst, B.; Kalache, A.; Shekhar, C.; Kumar, N.; Chadov, S.; Felser, C.; Parkin, S. S. P.; Nayak, A. K. Compensated Ferrimagnetic Tetragonal Heusler Thin Films for Antiferromagnetic Spintronics. Adv. Mater. 2016, 28, 8499-8504.

(32) Dyadkin, V.; Pattison, P.; Dmitriev, V.; Chernyshov, D. A New Multipurpose Diffractometer PILATUS@SNBL.J. Synchrotron Rad. 2016, 23, 825-829.

(33) Rodríguez-Carvajal, J. Recent Advances in Magnetic Structure Determination by Neutron Powder Diffraction. Physica B 1993, 192, 55-69. 
(34) Sears, V. F. International Tables for Crystallography, edited by A. J. C. Wilson (Kluwer Academic Publishers, Dordrecht/Boston/London, 1995), Vol. C, p. 383.

(35) Brown, P. J. International Tables for Crystallography, edited by A. J. C. Wilson (Kluwer Academic Publishers, Dordrecht/Boston/London, 1995), Vol. C, p. 391.

(36) Kobayashi, K.; Nagao, T.; Ito, M. Radial Integrals for the Magnetic Form Factor of $5 d$ Transition Elements. Acta Cryst. A 2011, 67, 473-480.

(37) http://mosswinn.com.

(38) Kim, S. H.; Battle, P. D. Structural and Electronic Properties of the Mixed Co/Ru Perovskites $A A^{\prime} \mathrm{CoRuO}_{6}\left(A, A^{\prime}=\mathrm{Sr}, \mathrm{Ba}, \mathrm{La}\right)$. J. Solid State Chem. 1995, 114, 174-183.

(39) Retuerto, M.; Alonso, J. A.; Martínez-Lope, M. J.; García-Hernández, M.; Krezhov, K.; Spiro, I.; Ruskov, T.; Fernández-Díaz, M. T. Crystal Structure and Magnetism of the $6 \mathrm{H}$ Hexagonal Double Perovskites $\mathrm{Ba}_{2} \mathrm{FeSbO}_{6}$ and $\mathrm{Ba}_{2} \mathrm{CoSbO}_{6-\delta}$ : A Neutron Diffraction and Mössbauer Spectroscopy Study. Eur. J. Inorg. Chem. 2008, 2008, 2286-2294.

(40) Flynn, J.; Li, J.; Sleight, A. W.; Ramirez, A. P.; Subramanian, M. A. Structure and Properties of Ir-containing Oxides with Large Spin-Orbit Coupling: $\mathrm{Ba}_{2} \mathrm{In}_{2-x} \mathrm{Ir}_{x} \mathrm{O}_{5+\delta}$. Inorg. Chem. 2016, 55, 2748-2754.

(41) Stitzer, K. E.; Abed, A. E.; Smith, M. D.; Davis, M. J.; Kim, S.-J.; Darriet, J.; zur Loye, H.-C. Crystal Growth of Novel Osmium-containing Triple Perovskites. Inorg.Chem. 2003, 42, 947-949.

(42) Mogare, K. M.; Klein, W.; Jansen, M, $\mathrm{K}_{2} \mathrm{NaOsO}_{5.5}$ and $\mathrm{K}_{3} \mathrm{NaOs}_{2} \mathrm{O}_{9}$ : The First Osmium Perovskite Containing Alkali Cations at the “A” Site. J. Solid State Chem. 2012, 191,153157.

(43) Morrow, R.; Samanta, K.; Dasgupta, T. S.; Xiong, J.; Freeland, J. W.; Haskel, D.; Woodward, P. M. Magnetism in $\mathrm{Ca}_{2} \mathrm{CoOsO}_{6}$ and $\mathrm{Ca}_{2} \mathrm{NiOsO}_{6}$ : Unraveling the Mystery of Superexchange Interactions between $3 d$ and $5 d$ Ions. Chem. Mater. 2016, 28, 3666-3675.

(44) Balcells, L. I.; Navarro, J.; Bibes, M.; Roig, A.; Martínez, B.; Fontcuberta, J. Cationic Ordering Control of Magnetization in $\mathrm{Sr}_{2} \mathrm{FeMoO}_{6}$ Double Peovskite. Appl. Phys. Lett. 2001, 78, 781-783.

(45) Michalik, J. M.; De Teresa, J. M.; Ritter, C.; Blasco, J.; Serrate, D.; Ibarra, M. R.; Kapusta, C.; Freudenberger, J.; Kozlova, N. High- Field Magnetization Measurements in $\mathrm{Sr}_{2} \mathrm{CrReO}_{6}$ Double Perovskite: Evidence for Orbital Contribution to the Magnetization. Europhys. Lett. 2007, 78, 17006.

(46) Sánchez, D.; Alonso, J. A.; García-Hernández, M.; Martínez-Lope, M. J.; Martínez, J. L. Origin of Neutron Magnetic Scattering in Antisite-Disordered $\mathrm{Sr}_{2} \mathrm{FeMoO}_{6}$ Double 
Perovskites. Phys. Rev. B: Condens. Matter Mater. Phys. 2002, 65, 104426.

(47) Meiklejohn, W. H.; Bean, C. P. New Magnetic Anisotropy. Phys. Rev. 1956, 102, 14131414.

(48) Giri, S.; Patra, M.; Majumdar, S. Exchange Bias Effect in Alloys and Compounds. J. Phys.: Condens. Matter 2011, 23, 073201.

(49) Sahoo, R. C.; Giri, S. K.; Dasgupta, P.; Poddar, A.; Nath, T. K. Exchange Bias Effect in Ferromagnetic $\mathrm{LaSrCoMnO}_{6}$ Double Perovskite: Consequence of Spin-Glass-Like Ordering at low Temperature. J. Alloys Compd. 2016, 658, 1003-1009.

(50) Nayak, A. K.; Nicklas, M.; Chadov, S.; Shekhar, C.; Skourski, Y.; Winterlik, J.; Felser, C. Large Zero-field Cooled Exchange-Bias in Bulk Mn ${ }_{2}$ PtGa. Phys. Rev. Lett. 2013, 110, 127204.

(51) Paul, A. K.; Sarapulova, A.; Adler, P.; Reehuis, M.; Kanungo, S.; Mikhailova, D.; Schnelle, W.; Hu, Z.; Kuo, C.; Siruguri, V.; Rayaprol, S.; Soo, Y.; Yan, B.; Felser, C.; Tjeng, L. H.; Jansen, M. Magnetically Frustrated Double Perovskites: Synthesis, Structural Properties, and Magnetic Order of $\mathrm{Sr}_{2} \mathrm{BOsO}_{6}(B=\mathrm{Y}, \mathrm{In}, \mathrm{Sc})$. Z. Anorg. Allg. Chem. 2015, 641, 197 205.

(52) Taylor, A. E.; Morrow, R.; Singh, D. J.; Calder, S.; Lumsden, M. D.; Woodward, P. M.; Christianson, A. D. Magnetic Order and Electronic Structure of the $5 d^{3}$ Double Perovskite $\mathrm{Sr}_{2} \mathrm{ScOsO}_{6}$. Phys. Rev. B 2015, 91, 100406 (R).

(53) Adler, P.; Ksenofontov, V.; Paul, A. K.; Reehuis, M.; Yan, B.; Jansen, M.; Felser, C. Magnetic Phase Transitions and Iron Valence in the Double Perovskite $\mathrm{Sr}_{2} \mathrm{FeOsO}_{6}$. Hyperfine Interact. 2014, 226, 289-297.

(54) Deng, H.; Liu, M.; Hu, Z.; Kuo, C.; Yin, Y.; Yang, J.; Wang, X.; Zhao, Q.; Xu, Y.; Fu, Z.; Cai, J.; Guo, H.; Jin, K.; Pi, T.; Soo, Y.; Zhou, G.; Cheng, J.; Chen, K.; Ohresser, P.; Yang, Y.-F.; Jin, C.; Tjeng, L. H.; Long, Y. Strong Enhancement of Spin Ordering by A-Site Magnetic Ions in the Ferrimagnet $\mathrm{CaCu}_{3} \mathrm{Fe}_{2} \mathrm{Os}_{2} \mathrm{O}_{12}$. Phys. Rev. B 2016, 94, 024414.

(55) Deng, H.; Liu, M.; Hu, Z.; Kuo, C.; Yin, Y.; Yang, J.; Wang, X.; Zhao, Q.; Xu, Y.; Fu, Z.; Cai, J.; Guo, H.; Jin, K.; Pi, T.; Soo, Y.; Zhou, G.; Cheng, J.; Chen, K.; Ohresser, P.; Yang, Y.-F.; Jin, C.; Tjeng, L. H.; Long, Y. Strong Enhancement of Spin Ordering by A-Site Magnetic Ions in the Ferrimagnet CaCu3Fe2Os2O12. Phys. Rev. B: Condens. Matter Mater. Phys. 2016, 94, 024414. 


\section{Graphical abstract}

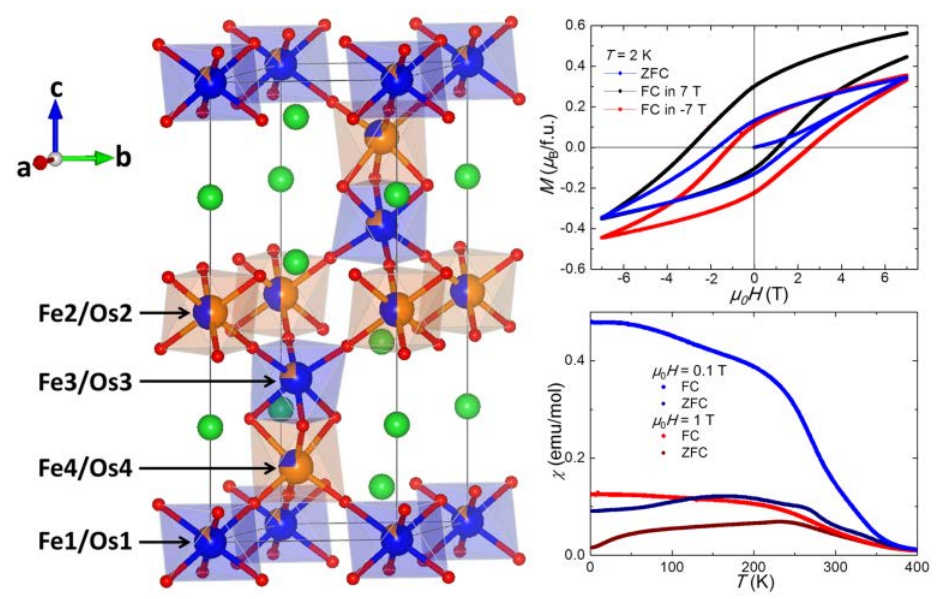

